Urologe 2009 · 48:1151

DOI 10.1007/s00120-009-2114-1

Online publiziert: 19. September 2009

(c) Springer Medizin Verlag 2009

\section{T. Kälble $\cdot$ O. Moormann ${ }^{2}$}

${ }^{1}$ Klinik für Urologie und Kinderurologie, Klinikum Fulda gAG, Fulda

${ }^{2}$ Fachklinik für Urologie, Kinderurologie, Andrologie und urologische Onkologie, Paracelsus-Klinik Golzheim, Düsseldorf

\title{
Akuter und chronischer Schmerz in der Urologie
}

Eine organisierte, individualisierte Schmerztherapie entsprechend der S3Leitlinien zur perioperativen Schmerztherapie ist längst nicht in allen urologischen Kliniken umgesetzt. Dabei ist das Vermeiden von beispielsweise postoperativen Schmerzen mit konsekutiver Katabolie bzw. sympathikoadrenergen $\mathrm{Zu}$ ständen ein wichtiger Baustein zur Senkung der perioperativen Morbidität, u. U. sogar der Mortalität. Besonders eklatant erscheint der Mangel an standardisierter Kinderanalgesie nach verschiedensten Operationen. Laut P. Reinhold (Herford) erfolgt nur bei $29 \%$ der in Deutschland operierten Kinder eine ausreichende Schmerztherapie.

Ein weiteres Spezifikum in der urologischen Schmerztherapie sind für Arzt und Patient oft unbefriedigende Symptome wie das chronische Beckenschmerzsyndrom, Postvasektomieschmerzen, Leisten- oder Hodenschmerzen mit und ohne Voroperationen etc.. Auch Frauen aus dem gynäkologisch/urologischen Grenzgebiet mit chronischen Becken- schmerzen gehören immer wieder zu unseren Patienten.

So versucht das vorliegende Heft mit Übersichtsarbeiten einschließlich einer eingereichten Originalarbeit praxisnah die verschiedenen Aspekte des akuten und chronischen Schmerzes in der Urologie abzuhandeln.

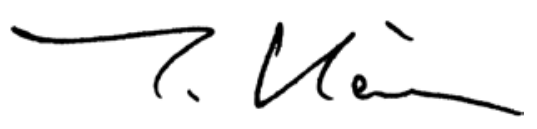

T. Kälble

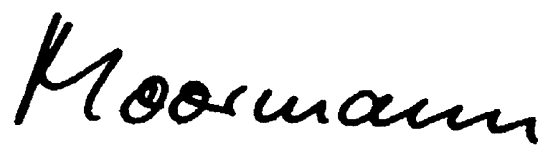

O. Moormann

\author{
Korrespondenzadresse \\ Prof. Dr. T. Kälble \\ Klinik für Urologie und Kinderurologie, \\ Klinikum Fulda gAG, \\ Pacelliallee 4, 36043 Fulda \\ T.Kaelble.Urologie@klinikum-fulda.de
}

